\title{
Lymphatic involvement in Chikungunya patient in Cardiovascular Practice
}

\author{
KHURSHED AHMED ${ }^{1}$, HARISUL HOQUE ${ }^{1}$, FAZLUR RAHMAN ${ }^{1}$, JAHANARA ARZU ${ }^{1}$, MANZOOR \\ MAHMOOD ${ }^{1}$, SM MUSTAFA ZAMAN ${ }^{1}$, SAJAL K BANERJEE ${ }^{1}$, SYED ALI AHSAN ${ }^{1}$, CHOUDHURY MESHKET \\ AHMED $^{1}$, SM AHSAN HABIB ${ }^{1}$, MD MUKHLESUR RAHMAN ${ }^{1}$, MD. SAIF ULLAH KHAN ${ }^{2}$, NILUFAR FATEMA ${ }^{1}$ \\ ${ }^{1}$ Department of Cardiology, Bangabandhu Sheikh Mujib Medical University (BSMMU), Dhaka, \\ ${ }^{2}$ Department of Vascular Surgery, BSMMU, Dhaka
}

Address for Correspondence: Dr. Khurshed Ahmed, Associate Professor, Department of Cardiology, BSMMU, Dhaka. Email:dmkahmed28@gmail.com

\begin{abstract}
:
Chikungunya virus (CHIKV) is an RNA alphavirus of the Togaviridae family that produces an acute febrile illness in humans followed by Joint pain, Itchy rash and leg swelling. This emerging virus has caused several large outbreaks in parts of Africa, Asia, and the Indian Ocean Islands and more recently in the Caribbean. This study was done from December 2015 to November 2016 on 24 confirmed Chikungunya patients with leg swelling. Peripheral vascular duplex study was done in every patients to find out the cause of leg swelling. Unilateral leg swelling $83 \%$ and Bilateral leg swelling $17 \%$. Lower limb vascular Duplex was done in all patients. Moderate resersible lymphatic oedema in subcutaneous tissue of lower limb was found in 22 patients only. 2 patients had cellulites with mild lymphatic swelling. DVT was absent. There was mild reduction of peak systolic arterial flow in 13 patients which is secondary to pressure effect of lymphedema and leg swelling. 16 patients had non tender lymphadenopathy (>1cm in diameter), 2 had tender lymphadenopathy in inguinal region and no enlarged lymph glands was observed in rest of the 6 patients. 6 patients had neutropenia and 8 had lymphopenia. Gradual improvement of symptoms was observed with conservative treatment. Lymphedema is reversible and conservative therapy is appropriate. And Non tender lymphadenopathy does not require treatment.
\end{abstract}

University Heart Journal 2017; 13(1): 13-16

Introduction:

Chikungunya virus (CHIKV) is an RNA alphavirus of the Togaviridae family. It produces an acute febrile illness, Joint pain, Itchy rash in humans ${ }^{1}$. This emerging virus has caused several large outbreaks in parts of Africa, Asia, the Indian Ocean Islands, Caribbean and more recently in the Bangladesh. More than 850,000 suspected and confirmed cases have been reported during this outbreak in the Caribbean and in South and Central American countries. Imported cases in returning travelers have also been diagnosed in non-endemic countries such as the United States, France, Italy, england and Spain ${ }^{2}$. More than $75 \%$ of human CHIKV infections are symptomatic ${ }^{1}$. Lymphadenopathy has been described in infections due to other alphaviruses such as O'nyong-nyong virus 3; yet, it is not a commonly reported feature in CHIKV infections. First 6 case reports of Lymphaadenopathy of Chikungunya have been published by Francesca F. Noman et.al. ${ }^{3}$ Another case report of painful cervical lymphadenopathy as an unusual presentation of Chikungunya published by Mukundraj S Keny et.al. ${ }^{4}$ In one study the vascular specialists had done the diagnostic work up of leg swelling( by a pain-free duplex scanning. ${ }^{5}$

Aim of the study: To find out the cause of leg swelling in Chikungunya patients.
Methods:

- Study type: Observational study

- Study population: total 24 Chikungunya patients with leg swelling who underwent lower limb vascular duplex study

- Study period: December 2015 to November 2016

- Study place: Labaid, Uttara, Dhaka, Bangladesh

Inclusion criteria:

- Chikungunya patients with leg swelling.

- Chikungunya IgG and IgM positive.

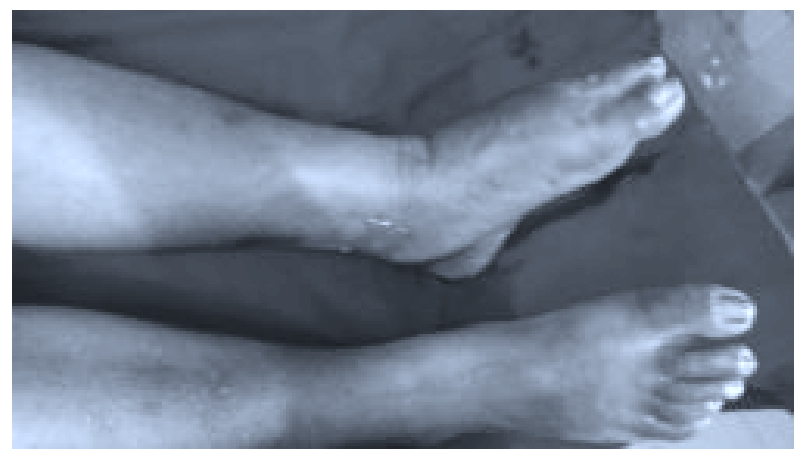

Fig.-1: 


\section{Exclusion criteria:}

- Patient with heart failure

- Patient with renal failure

- Patient with liver failure

A questionnaire was used for each patient. Data were analyzed by SPSS 19.0 software.

\section{Results:}

This study included 24 Chikungunya patients. Initially fever, joint pain, most presented with rash were observed in these patients. Leg swelling was apparent at the end or after fibrile period. Small joints were involved with symmetrical pattern. Regarding age, it was observed that, age ranged from 18-60 years. All patients were Chikungunya IgG and IgM positive. Unilateral leg swelling $83 \%$ and Bilateral leg swelling $17 \%$. Lower limb vascular Duplex was done in all patients. Moderate resersible lymphatic oedema in subcutaneous tissue of lower limb was found in 22 patients only. 2 patients had cellulites with mild lymphatic swelling. DVT was absent. There was mild reduction of peak systolic arterial flow in 13 patients which is secondary to pressure effect of lymphedema and leg swelling. 16 patients had non tender lymphadenopathy ( $>1 \mathrm{~cm}$ in diameter), 2 had tender lymphadenopathy in inguinal region and no enlarged lymph glands was observed in rest of the 6 patients. 6 patients had neutropenia and 8 had lymphopenia. Gradual improvement of symptoms was observed with conservative treatment.

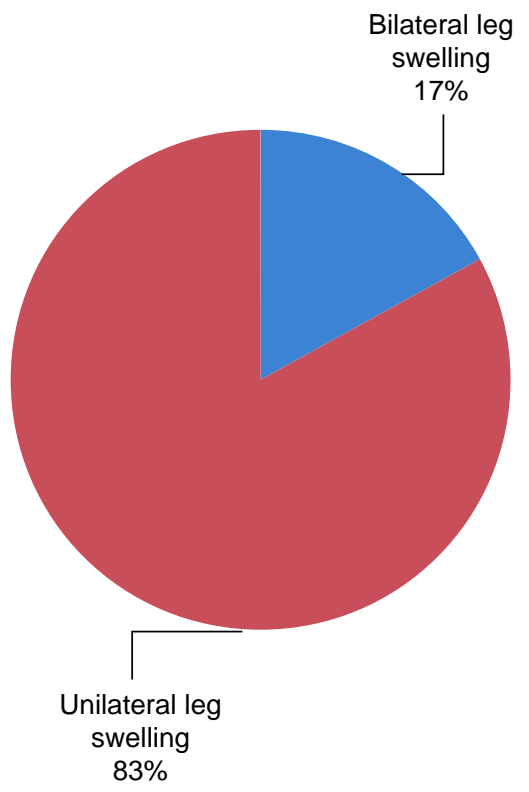

Fig.-2: Unilateral leg swelling $83 \%$ and Bilateral leg swelling $17 \%$

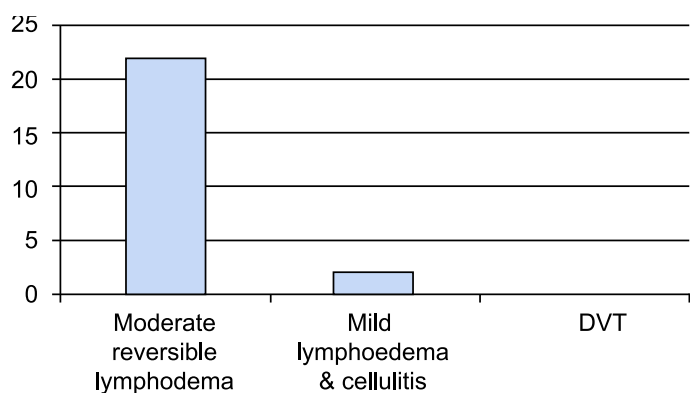

Fig.-3: Duplex scan of lower limb revealed :1) Moderately reversible lymphatic oedema in subcutaneous tissue of lower limb was found in 22 patients. 2) Only 2 patients had cellulitis with mild lymphatic swelling. 3) DVT was absent.

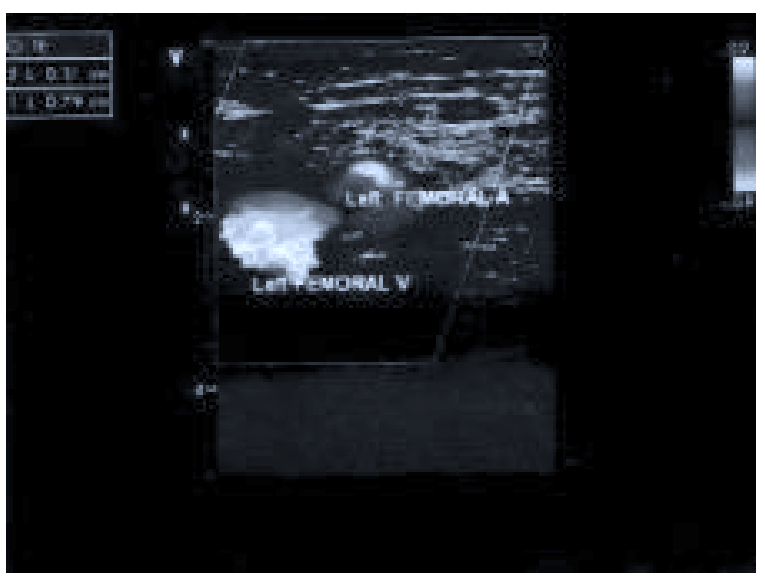

Fig.-4: Lymphadenopathy lateral to femoral artery in study subject

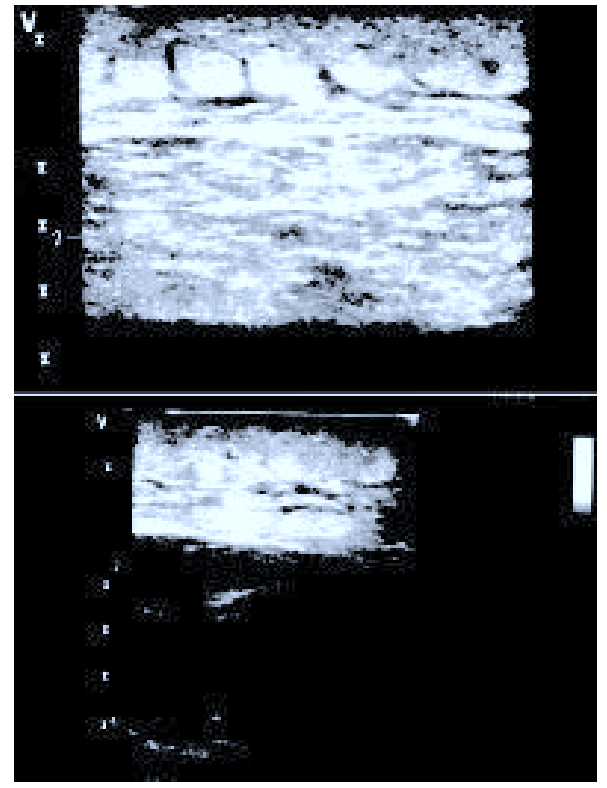

Fig.-5: Lymphatic swelling in subcutaneous tissue 


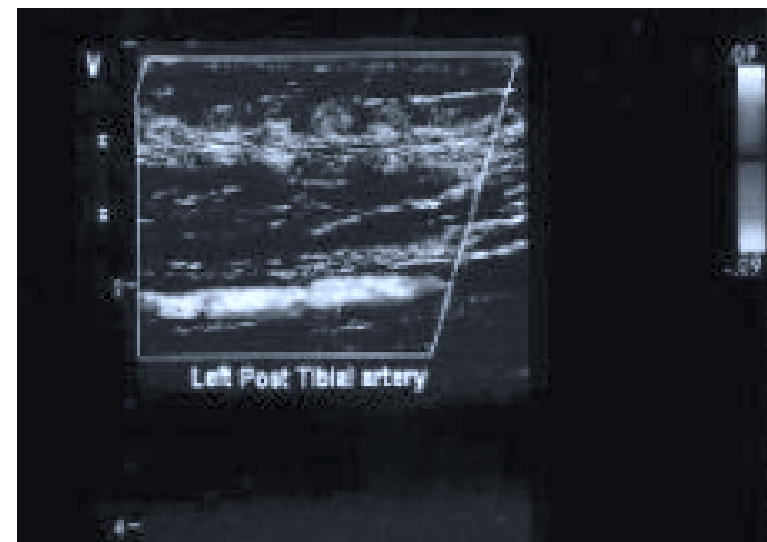

Fig.-6: Posterior tibial artery signal found deep seated due to superficial lymphatic swelling

\section{Discussion:}

CHIKV is currently spreading in the Bangladesh for last two- three years. The surveillance of clinical infections in this new geographical area is essential. Although lymphadenopathy has previously been described in the context of alphavirus infections, this is an uncommonly reported feature in infections caused by CHIKV. Lymphadenopathy, mainly of the cervical area, was reported in nearly half of the patients during an outbreak of O'nyong-nyong fever in south-central Uganda in the late 1990s. ${ }^{6}$ However, in the case series of CHIKV infection outbreak in La Réunion in 2006, lymphadenopathy was reported frequently, where lymphadenopathy was found in $<9 \%$ of 157 of the confirmed cases described. ${ }^{7}$ In another study conducted in India in 2006, lymphadenopathy was present in around $14 \%(12 / 87)$ of confirmed cases. ${ }^{8}$ Two other case series have reported lymphadenopathy in $18 \%$ of 28 acute CHIKV infections diagnosed at a hospital in Southern Sri Lanka in 2007 and pre- and post-auricular lymphadenopathy in 4 of 220 suspected CHIKV cases in an area of East India in 2011, respectively. ${ }^{9,10}$ The clinical spectrum of the infection may be different in pediatric cases as lymphadenopathy has been described to be a common clinical feature in a review of CHIKV in children. ${ }^{11}$ In this study of the current outbreak in Bangladesh revealed that nontender lymphadenopathy was present in more than half of the cases. Although more data will become available as additional information from this evolving outbreak is recorded, it appears that lymph node involvement in the context of CHIKV infections and lymph oedema may be more frequent than previously determined. It is also possible that the occurrence of lymphadenopathy and lymph oedema may be underdiagnosed given that it may occur toward the end of the febrile period during recovery from the infection and appear to be self-limiting. This feature may therefore not be recorded unless it is self-reported. The possibility that more pronounced symptoms such as painful reactive lymphadenopathy may be more frequent during the current outbreak as a new alphavirus has been recently introduced into an apparently population should be explored. Some studies have examined the possible role of RNA viruses in the pathogenesis of Burkitt's lymphoma, and CHIKV has been investigated as a potentially oncogenic arbovirus. ${ }^{12,13}$ In addition, models of chikungunya infection in nonhuman primates have found long-term persistence of CHIKV in joints, muscles, lymphoid organs, and liver. ${ }^{14}$ The possible longterm consequences of this infection in such a population should therefore be determined. Other acute infections that could lead to a similar clinical presentation with fever and lymphadenopathy such as EBV, CMV, Toxoplasma sp., or HIV infections were not excluded in all cases in this study. The combination of clinical, epidemiological, and virological criteria for CHIKV infection in all the cases made other etiologies less likely. Patients with suspected CHIKV infection should be examined for the presence of lymphadenopathy. Further studies would be needed on immunological factors or biomarkers of infection in those patients presenting with lymphadenopathy may be the future aid of understanding of the pathogenesis of this viral infection in humans.

\section{Conclusion:}

Chikungunya patient who have leg swelling can be done lower limb vascular duplex to exclude DVT, may assure that lymphedema is reversible and conservative therapy is appropriate. And Non tender lymphadenopathy does not require treatment.

\section{References:}

1. Burt FJ, Rolph MS, Rulli NE et al. Chikungunya: a re-emerging virus. Lancet 2012; 379:662-71.

2. European Centre for Disease Prevention and Control. Communicable Disease Threats Report. 16-22 November 2014. http://www.ecdc.europa.eu/en/publications/ Publications/ communicable-disease-threats-report-22-nov-2014.pdf . (Accessed 2014 Dec 3)

3. Francesca F. Noman et al. Lymphadenopathy in Patients With Chikungunya Virus Infection Imported From Hispaniola: Case Reports. J Travel Med 2015;22:272-75

4. Mukundraj S Keny, Ian A Percira et al. Painful cervical lymphadenopathy: An unusual presentation of Chikungunya. Int J Appl Basic Med Res. 2014 Jan-Jun:4(1): 47-49

5. Work up of lymphatic disease, by 'Vascular institute Switzerland'. www.angiologie-aargau.ch/en/services/lymph/ 
6. Kiwanuka N, Sanders EJ, Rwaguma EB, et al. O'nyong-nyong fever in south-central Uganda, 1996-1997: clinical features and validation of a clinical case definition for surveillance purposes. Clin Infect Dis 1999; 29:1243-50.

7. Borgherini G, Poubeau P, Staikowsky F, et al. Outbreak of chikungunya on Reunion Island: early clinical and laboratory features in 157 adult patients. Clin Infect Dis 2007; 44:1401-07.

8. Suryawanshi SD, Dube AH, Khadse RK, et al. Clinical profile of chikungunya fever in patients in a tertiary care centre in Maharashtra, India. Indian J Med Res 2009; 129:438-41.

9. Reller ME, Akoroda U, Nagahawatte A, et al. Chikungunya as a cause of acute febrile illness in Southern Sri Lanka. PLoS One 2013; 8:e82259.
10. Gurav YK, Gopalkrishna V, Shah PS, et al. An outbreak of chikungunya in Jamshedpur, Jharkhand in 2011. Indian J Med Res 2012; 136:886-89.

11. Sebastian MR, Lodha R, Kabra SK. Chikungunya infection in children. Indian J Pediatr 2009; 76:185-89.

12. van den Bosch C, Lloyd G. Chikungunya fever as a risk factor for endemic Burkitt's lymphoma in Malawi. Trans R Soc Trop Med Hyg 2000; 94:704-05.

13. van den Bosch C. A role for RNA viruses in the pathogenesis of Burkitt's lymphoma: the need for reappraisal. Adv Hematol 2012; 2012:494758.

14. Labadie K, Larcher T, Joubert C, et al. Chikungunya disease in nonhuman primates involves long-term viral persistence in macrophages. J Clin Invest 2010; 120:894-906. 